\title{
REDUCED-ORDER MODELS BASED ON LINEAR AND NONLINEAR AERODYNAMIC IMPULSE RESPONSES
}

\author{
Walter A. Silva* \\ Aeroelasticity Branch \\ NASA Langley Research Center
}

$$
\begin{aligned}
& 53 / .02 \\
& 381333 \\
& p .
\end{aligned}
$$

\section{ABSTRACT}

This paper discusses a method for the identification and application of reduced-order models based on linear and nonlinear aerodynamic impulse responses. The Volterra theory of nonlinear systems and an appropriate kemel identification technique are described. Insight into the nature of kernels is provided by applying the method to the nonlinear Riccati equation in a non-aerodynamic application. The method is then applied to a nonlinear aerodynamic model of an RAE 2822 supercritical airfoil undergoing plunge motions using the CFL3D Navier-Stokes flow solver with the Spalart-Allmaras turbulence model. Results demonstrate the computational efficiency of the technique.

\section{INTRODUCTION}

As the complexity of modern computational fluid dynamics (CFD) codes increases, so does their computational cost and execution time. As a result, these codes are not used routinely in disciplines where the information provided by these codes could be of great benefit. These disciplines include aeroelasticity, aeroservoelasticity, optimization, and preliminary design. In order to improve this situation, the development of reduced-order models has become a major goal of several national and international organizations $^{1-12}$.

A reduced-order model is a simplified mathematical model that encapsulates most, if not all, of the fundamental dynamics of a more complex system. Due to its mathematical simplification, the computational cost (CPU *Research Scientist, Senior Member Copyright(C) 1999 by the American Institute of Aeronautics and Astronautics, Inc. No copyright is asserted in the United States under Title 17. U.S.Code. The U.S. Government has a royaltyfree license to exercise all rights under the copyright claimed herein for Governmental Purposes. All other rights are reserved by the copyright owner. memory, execution time, and turnaround time) of using a reduced-order model can be orders of magnitude lower than the computational cost of using the original more complex system. In the case of CFD codes, development of aerodynamic reduced-order models provides a cost-effective means for incorporating CFD analyses into several disciplines where, heretofore, it has not been incorporated.

This paper will discuss reduced-order aerodynamic models based on linear and nonlinear aerodynamic impulse responses. Previously ${ }^{9-12}$, the concept of an aerodynamic impulse response was introduced and its relationship to the more traditional aerodynamic functions (Wagner's, Theodorsen's) was defined. Aerodynamic impulse responses are obtained from any CFD model of interest using standard digital signal processing techniques and the Volterra theory of nonlinear systems $^{y-12}$. Computationally-efficient linear and nonlinear digital convolution schemes are then applied for predicting the response of the nonlinear aerodynamic system to arbitrary inputs.

The paper begins with mathematical definitions of time-invariant and time-varying systems. This is followed by a description of the Volterra theory of nonlinear systems, including derivation of the kernel identification equations. These kernel identification equations are then applied to nonlinear systems in order to gain insight into the nature of the kernels. The nonlinear systems investigated include: first, a nonlinear Riccatti circuit which will illustrate the nature of kernels and then a plunging airfoil using the CFL3D (Navier-Stokes) flow solver with the Spalart-Allmaras turbulence model.

\section{MATHEMATICAL SYSTEMS}

A time-invariant (TI) system, also referred to as a shift-invariant, stationary or autonomous system, is a system whose fundamental properties do not change with time. That is, the equations defining a TI system are not explicit functions of time so 


$$
f=f(x, \dot{x}, \ddot{x}, \ldots)
$$

An example of a simple, TI, nonlinear system is a pendulum. Although the full nonlinear equation of a pendulum is certainly a function of time which can exhibit nonlinear, unsteady responses if an unsteady excitation is applied, neither the length of the pendulum nor the mass at the end of the pendulum are functions of time $e^{13}$

Differential equations with constant coefficients are TI because the coefficients are not explicit functions of time. But not all TI systems are defined by equations with constant coefficients. Time-invariance is sometimes mistakenly interpreted as implying functions that are independent of time. Even a classical, fundamental text such as Ref. 14 misinterprets nonlinear, TI systems as systems that do not accept time-dependent forcing functions. This is clearly not correct since the time-invariance of a system refers to the system itself and not to the characterization of the inputs or outputs (i.e., steady or unsteady) of the system.

A time-varying (TV) system, also referred to as a non-stationary or non-autonomous system, is a system whose fundamental properties do change with time. That is

$$
f=f(x, \dot{x}, \ddot{x}, \ldots, t)
$$

An example of a TV system is a rocket during launch. The mass of the rocket, mostly fuel, is spent very quickly. The mass of the rocket, and therefore the rocket's dynamics, are changing with time. The identification of impulse responses for a time-varying system is typically more complicated than for a TI system. Reference 15 addresses the problem of Volterra kernel identification for TV, nonlinear systems.

Fortunately, for many of the problems in aircraft unsteady aerodynamics, aeroelasticity, and aeroservoelasticity, the governing nonlinear equations are time invariant. Although an airplane's fuel quantity, or mass, is certainly not constant, present-day analyses treat an airplane's fuel loading as separate, constant-mass cases (full fuel to near empty, for example) as opposed to a continuously-varying quantity. The linearization of these TI, nonlinear equations about an operating point yields the familiar TI, linear equations that comprise the majority of modem- day, linear analysis techniques within these disciplines.

The Navier-Stokes equations do not have any coefficients that are explicit functions of time. As a result, the Navier-Stokes equations are, by definition, time invariant ${ }^{11.12}$. The discretized Navier-Stokes equations, or CFD codes, do exhibit time-varying behavior at certain conditions, especially in the initial time steps when the residual (error) is in the process of converging to an acceptably small number. But the residual term is a byproduct of discretization that is not present in the continuous-time NavierStokes equations. A condition of consistency requires that, in the limit, the discretized system approach the original, continuous-time system. Therefore, given appropriate discretization and convergence of the residual, the discretized Navier-Stokes equations should be, and are in fact, TI as well. This is important since it would be unacceptable for a discretization process to transform a TI system into a TV system, as the associated dynamics of a TI system versus those of a TV system are vastly different.

\section{VOLTERRA THEORY}

\section{Introduction}

The Volterra ${ }^{16}$ theory was developed in 1930. The theory is based on functionals, or functions of other functions, and subsequently became a generalization of the linear convolution integral approach that is applied to linear, time-invariant (LTI) systems.

The basic premise of the Volterra theory of nonlinear systems $s^{17.18}$ is that any nonlinear system can be modeled as an infinite sum of multidimensional convolution integrals of increasing order. This infinite sum, presented here in continuous-time form, is known as the Volterra series and it has the form

$$
\begin{aligned}
& y(t)=h_{0}+\int_{0}^{\infty} h_{1}(t-\tau) u(\tau) d \tau+ \\
& \int_{0}^{\infty} \int_{0}^{\infty} h_{2}\left(t-\tau_{1}, t-\tau_{2}\right) u\left(\tau_{1}\right) u\left(\tau_{2}\right) d \tau_{1} d \tau_{2}+\ldots \\
& +\int_{0}^{\infty} \ldots \int_{0}^{\infty} h_{n}\left(t-\tau_{1} \ldots . . t-\tau_{n}\right) u\left(\tau_{1}\right) \ldots u(m) d \tau_{1} \ldots d \tau_{n}+\ldots
\end{aligned}
$$


where $y(t)$ is the response of the nonlinear system to $u(t)$, an arbitrary input; $h_{0}$ is a steady value about which the response is computed; $h_{1}(t)$ is the first-order kernel or the linear unit impulse response; $h_{2}\left(\tau_{1}, \tau_{2}\right)$ is the second-order kernel, and $h_{n}\left(\tau_{1}, \ldots, \tau_{n}\right)$ is the $n^{\text {th }}$-order kernel. It is assumed that: 1) the kernels, input function, and the output function are real-valued functions; 2) the system is causal ; and 3 ) the system is time invariant.

Inspection of Equation (1) reveals some very interesting and characteristic features of the Volterra series. The value of $h_{0}$ is known based on the steady-state value of the system at a particular condition. It does not require any special identification technique. This will be discussed in more detail when applied to an aerodynamic system. Also, if the kernels of order two and above are zero, then the response of the system is linear and is completely described by the unit impulse response $h_{1}(t)$, and the firstorder convolution integral.

The higher order kernels $\left(h_{2}\left(\tau_{1}, \tau_{2}\right), \ldots\right.$, $\left.h_{n}\left(\tau_{1}, \ldots, \tau_{n}\right)\right)$ are the responses of the nonlinear system to multiple unit impulses, with the number of impulses applied equal to the order of the kernel of interest : e.g., $h_{2}\left(\tau_{1}, \tau_{2}\right)$ is the response of the nonlinear system to two unit impulses applied at two points in time, $\tau_{1}$ and $\tau_{2}$. The variation of the time difference between these two times characterizes the second-order (nonlinear) memory of the system. Therefore, the second-order kernel is a two-dimensional function of time: $t$ and the time difference $T=\tau_{1}$ $\tau_{2}$. This mathematical definition follows directly for the $n^{\text {th }}$-order kernel, although visualization of these functions can become difficult for orders greater than three. As will be shown, these kernels are also a function of the amplitude of the input used for identification.

The impulse response of a linear system is referred to as the memory of the system. Convolution then allows exact prediction of the response of the system to an arbitrary input because all responses of the system are scaled and shifted superpositions of this memory function ${ }^{12}$. It is important to understand that the set of arbitrary inputs includes any and all possible inputs, from steady (step) inputs to random inputs, thus the term "arbitrary". For the linear case, the arbitrary input has no amplitude or frequency limitations.
For a nonlinear system approximated by a Volterra series, the higher-order kernels are a measure of the nonlinear memory of the system. Unlike the linear system, however, the arbitrary nature of the input, primarily with respect to amplitude, does have some limitations due to the fact that the Volterra series is truncated for practical applications. As Boyd ${ }^{19}$ has shown, the convergence of the Volterra series is limited by the infinity norm of the input (maximum value). If this norm exceeds a particular value, then convergence of the series, and, therefore, the predictive ability of the series, is not guaranteed. The infinity norm of the input is, of course, system dependent and will not usually be known a priori. Similarly, the convergence of the series is a function of the number of components that are identified for a particular kernel. Rugh ${ }^{17}$ and Boyd $^{19}$ discuss Volterra's (and Frechet's) extension of the Weierstrass theorem to nonlinear systems with finite (or fading) memory, and its relationship to the Voltera series.

Wiener ${ }^{20}$ contributed significantly to the development of the Volterra theory and, as a result, the theory is sometimes referred to as the Volterra-Wiener theory of nonlinear systems. Reference 21 presents a kernel identification technique based on auto- and cross-correlation functions. References 22-29 are additional, excellent sources of information regarding the Volterra theory of nonlinear systems.

This research focuses on the time-domain Volterra theory because CFD analyses are typically performed in the time domain. There exists, however, a great deal of information on the frequency-domain Volterra theory $y^{17.18 .30}$. The frequency-domain Volterra theory deals with the multidimensional Fourier transforms of the timedomain kernels. The resultant functions are referred to as higher-order spectra $a^{31,32}$. A double Fourier transform of a second-order kernel is referred to as a bispectrum. Whereas timedomain Volterra kernels may be better suited for computational methods, the frequency-domain methods appear to be better suited for experimental identification techniques. Boyd et al $^{33}$ describe a frequency-domain technique that was successfully applied to the experimental identification of the second-order kernel of a nonlinear electroacoustic transducer (speaker) system. The theory also has some very interesting applications in the fields of general turbulence ${ }^{\text {it }}$ and low-frequency drift oscillations (LFDO) experienced by moored vessels in 
turbulent seas ${ }^{35}$. A time-domain Volterra kernel identification technique is described in a subsequent section.

\section{Weakly Nonlinear Systems}

One approach for obtaining Volterra series representations of physical systems is to assume that the system is a 'weakly' nonlinear system. A weakly nonlinear system is well defined by the first two kernels of the Volterra series so that kernels of third order and above are negligible. Boyd, Tang, and Chua ${ }^{33}$ mention some physical systems that are accurately modeled as weakly nonlinear systems including electromechanical and electroacoustic transducers and some biological systems. In this study, it is assumed that the nonlinear aerodynamic system that is identified from the Navier-Stokes equations is a weakly nonlinear, second-order system. It is important to develop expertise with the application of Volterra methods to nonlinear aerodynamic models in a systematic manner and a weakly-nonlinear model provides this type of gradual approach to the problem.

Although this truncation may exacerbate known convergence and amplitude restrictions of the Volterra series, it is of interest to investigate the effectiveness of this truncated model to practical applications. The truncated, secondorder Volterra series is

$$
\begin{aligned}
& y(t)=h_{0}+\int_{0}^{\infty} h_{1}(t-\tau) u(\tau) d \tau+ \\
& \int_{0}^{\infty} \int_{0}^{\infty} h_{2}\left(t-\tau_{1}, l-\tau_{2}\right) u\left(\tau_{1}\right) u\left(\tau_{2}\right) d \tau_{1} d \tau_{2}
\end{aligned}
$$

For the applications considered in this study, kernel identification will consist of the identification of the first- and second-order kernels with $h_{1}$ clearly stated as appropriate.

\section{Kernel Identification}

The advantage of the Volterra series approach for modeling nonlinear systems is that once the kernels are identified, the response of the nonlinear system to an arbitrary input can be predicted. The problem of kernel identification, therefore, is central to the successful generation of an accurate Volterra series representation of a nonlinear system. The most obvious approach for identifying the kernels is to derive analytical expressions for the kernels from the governing nonlinear equations of the system of interest ${ }^{20.22 .23}$. Although this approach is theoretically applicable to any set of nonlinear equations, including the nonlinear fluid flow equations such as TSD, Euler, and Navier-Stokes equations, it would require a significant amount of effort to analytically compute the kernels for different configurations and for various inputs. Instead, a kernel identification technique is desired that uses the output of a CFD model directly for quick and efficient kernel identification, regardless of the CFD code being used and the particular model geometry.

In Eq. (2), analytical application of unit impulses (Dirac delta functions) results in equations that define the first- and second-order kernels. The equations are derived in detail in Ref. 12 and are presented here in final form:

$$
\begin{gathered}
h_{1}\left(\tau_{1}\right)=2 y_{0}\left(\tau_{1}\right)-(1 / 2) y_{2}\left(\tau_{1}\right) \\
h_{2}\left(\tau_{1}, \tau_{2}\right)=(1 / 2)\left(y_{1}\left(\tau_{1}, \tau_{2}\right)-y_{0}\left(\tau_{1}\right)-y_{0}\left(\tau_{2}\right)\right)
\end{gathered}
$$

where $y_{0}\left(\tau_{1}\right)$ is the response of the nonlinear system to a single unit impulse applied at time $\tau_{1} ; y_{0}\left(\tau_{2}\right)$ is the response of the nonlinear system to a single unit impulse applied at time $\tau_{2}$; $y_{1}\left(\tau_{1}, \tau_{2}\right)$ is the response of the nonlinear system to two unit impulses, one at time $\tau_{1}$ and one at time $\tau_{2}$; and $y_{2}\left(\tau_{1}\right)$ is the response of the nonlinear system to a single impulse at time $\tau_{1}$ (same time as $y_{0}\left(\tau_{1}\right)$ ) but with double the amplitude. For a TI system, $y_{0}\left(\tau_{2}\right)$ is just $y_{0}\left(\tau_{1}\right)$ shifted in time to $\tau_{2}$. It should be noted that $\tau_{1}$ is held constant (usually at $t=0$ ) while $\tau_{2}$ is varied for the computation of $y_{1}\left(\tau_{1}, \tau_{2}\right)$.

The first-order kernel, $h_{1}\left(\tau_{1}\right)$, is a onedimensional function of time. Clearly, for a purely linear system, the first-order kernel is identical to the linear unit impulse response. For a nonlinear system, the first-order kernel captures a first-order, amplitude-dependent deviation from linearity (i.e., nonlinearity) because it is comprised of two impulse responses of different amplitudes.

Once the first-order kernel has been computed, computation of the second-order kernel requires computation of several $y_{1}\left(\tau_{1}, \tau_{2}\right)$ responses for varying values of $\tau_{2}$. As a result, the second-order kernel is a two-dimensional function of time. It is a function of time $t$ and a 
function of the time difference between $\tau_{1}$ and $\tau_{2}$. As the time difference between $\tau_{1}$ and $\tau_{2}$ is varied, this leads to several values of $y_{1}\left(\tau_{1}, \tau_{2}\right)$ that, in turn, leads to several values of the second-order kernel. These responses are hereby referred to as "components" of the second-order kernel. Subsequent examples will clarify this concept.

\section{EXAMPLE- NONLINEAR CIRCUIT}

A simple nonlinear system that can be used to illustrate the kernel identification technique is a series circuit consisting of a linear inductance, a nonlinear resistance, and a voltage source ${ }^{28}$, shown in Figure 1. The governing equation for this circuit is the Riccati equation

$$
\frac{d y}{d t}+\alpha y+\varepsilon y^{2}=\alpha(t)
$$

with $y(t)$ the current around the circuit, $x(t)$ the input voltage, and $\alpha$ and $\varepsilon$ parameters from the nonlinear resistance. After discretization of the Riccati equation, the first-order kernel and several components of the second-order kernel, for this system, are generated using a time step of 0.01 . A time lag (difference between $\tau_{1}$ and $\tau_{2}$ ) of $T=$ 0.01 (or one time step) is used for computation of the components of the second-order kernel. The first component of the second-order kernel corresponds to $\mathrm{T}=0.0$ with both impulses at same point in time; the second component corresponds to $\mathrm{T}=0.01$ (one time step apart); the third component corresponds to $\mathrm{T}=0.02$ (two time steps apart); and so on.

Two cases wil be investigated. In the first, $\alpha=1.0$ and $\varepsilon=0.0001 ;$ in the second, $\alpha=0.1$ and $\varepsilon=0.001$. The effect of these variations on the nonlinearity of the system and the resultant first- and second-order kernels is investigated. For this system, the constant term in Eq. (2) $\left(h_{1}\right)$ is zero.

Case 1: $\alpha=1.0, \varepsilon=0.0001$

The first-order kernel for this case is presented in Figure 2 for 1000 time steps. Selected components for the corresponding second-order kernel are presented in Figure 3. Shown in Figure 3 are the first component, the one-hundred-and-first component, and so on. As can be seen, the largest component of the secondorder kernel (the tirst) is very much (seven orders of magnitude) smaller than the first-order kernel (Fig. 2) and goes to zero in about half the time. As might be expected with $\varepsilon=0.0001$, Figure 3 indicates that nonlinear effects for this case are quite small. Verification of this is presented in Figure 4, a comparison of various step responses obtained directly from the numerical solution of the Riccati equation (actual) and those obtained from the convolution of the step inputs with the first-order kernel of Figure $2 . \quad$ These indistinguishable results indicate that the firstorder kernel is sufficient to capture the response of this system for the range of amplitudes investigated.

Case 2: $\alpha=0.1, \varepsilon=0.001$

The first-order kernel for this case is presented in Figure 5, along with the first-order kernel from Case 1 (Fig. 2) for comparison purposes. The net effect of the change in the two parameters results in an increased effect of the nonlinearity of the Riccati equation. This is evidenced by the increased memory of the firstorder kernel (slower approach to zero) as compared with the first-order kernel of Case 1. Selected components from the second-order kemel for this case are presented in Figure 6, revealing a kernel two orders of magnitude larger than the second-order kernel of Case 1 (Fig. 3). Figure 7 is a comparison of step responses obtained directly from numerical solution of the Riccati equation (actual) and those obtained via convolution of the step inputs with the first-order kernel of Figure 5 for this system. A noticeable difference between step responses, as step amplitude is increased, indicates the effect of increased nonlinearity in the system and the need for the second-order kernel.

The sign of the second-order kernel is important since it is an indication of the effect of the second-order nonlinearity on the total response of the system. That is, since the second-order kernel of Figure 6 is negative, then the effect of the second-order convolution, which provides the effect of the second-order kernel, is to decrease the magnitude of the total response of the system from that oblained from the first-order convolution alone. This is clear in Figure 7, which shows that the response due to the firstorder term "overshoots" the actual response. Addition of the negative second-order response to the first-order response would cause the sum (both terms in Eq. (2)) to approach the actual response. The second-order kernel can therefore 
provide an indication of the additive effect of the second-order nonlinearity with respect to the firstorder term. The additional accuracy achieved, due to the inclusion of the second-order convolution, for the viscous Burger's equation has been demonstrated ${ }^{11.12}$ but is not presented here.

This example demonstrates the identitication of tirst- and second-order kernels of a simple nonlinear system. Inspection of the kernels can provide very useful information regarding the level of nonlinearity as well as the net effect of the nonlinearity of a particular system. These techniques will now be applied to a CFL3D model.

\section{RESULTS USING THE CFL3D CODE}

The CFL3D code ${ }^{36.37}$ (version 5.0) solves the time-dependent, Reynolds-averaged Navier-Stokes equations in conservation law form. Upwindbiasing is used for the pressure and convective terms, central differencing is used for the shear stress and heat transfer terms, and the spatial discretization is based on a semi-discrete finitevolume concept. Accelerated convergence can be achieved using multigrid and mesh sequencing capabilities and implicit time-stepping is used. The code provides several turbulence models, including the Spalart-Allmaras turbulence model used in the subsequent analyses.

\section{Results for RAE Airfoil}

Navier-Stokes results for a dense-grid RAE 2822 airfoil $^{36}$ with the Spalart-Allmaras turbulence model undergoing plunge at a Mach number of 0.75 , Reynold's number of 6.2 million, and a zero degree angle of attack were computed using a time step of 0.001 . At this condition, this non-symmetric supercritical airfoil induces a net normal force coefficient of 0.2953 . This corresponds to the $h_{v}$ term in Eq. (2). When generating the first- and second-order kernels for this system, $h_{v}$ has to be subtracted from the kernel computations. The response to a particular input is computed using the convolution procedures and then the $\mathbf{h}_{0}$ (= 0.2953 ) term is added back to obtain the total response.

The CFL3D code has several computational options, depending on the lype of analysis desired. Accelerated convergence can be obtained using the sub-iteration and multigrid capabilities $^{37}$. In addition, a method is available that diagonalizes the governing matrices (diagonally dominant) based on the spectral radius. Limited experimentation with these techniques, including the effects of tirst-order-intime versus second-order-in-time numerical accuracies are presented and discussed in Ref. 12. An optimal procedure for using multigrid and diagonalization to identify kernels has not yet been developed. As a result, the remainder of the results presented in this section are limited to solutions corresponding to second-order-in-time accuracy with no multigrid and no diagonalization.

These first- and second-order-in-time solutions refer to the numerical algorithm within CFL3D and should not be confused with first- and secondorder kernel functions.

Recall that the first-order kernel is identified using a response due to a unit plunge amplitude and a second response due to double that amplitude. An important question is "What is the effect of varying these amplitudes on the identification of the kernels and on their predictive capability?" Figure 8 is a comparison of non-diagonalized, no-multigrid, second-order accurate-in-time first-order kernels for two different identification input plunge amplitudes. The small-amplitude kernel of Figure 8 was identified using the primary amplitude of 0.01 and a secondary (doubled) amplitude of 0.02 . The large-amplitude kernel was identified using the primary amplitude of 0.1 and a secondary amplitude of 0.2 . The correlation between these two first-order kernels (Fig. 8) is not linear, as expected. That is, one kernel is not exactly ten times the other, indicating a deviation from linearity or some measure of nonlinearity. Therefore, for small amplitudes (linear regime), the first-order kernel is identical to a linearized (small perturbation) impulse response. At larger amplitudes, however, the furst-order kernel can capture a certain level of nonlinearity. It is important to note how quickly these first-order kernels reach equilibrium (go back to zero). This quick return to zero provides significant computational efficiency when extracting these functions from a CFD model, as will be seen.

The first five components of the second-order kernel tor this airfoil in plunge are presented in Figure 9. The input amplitude used to identify these components of the second-order kernel was 0.10 , consistent with the large-amplitude firstorder kernel of Figure 8 . Even so, the first component of the second-order kernel is an order 
of magnitude smaller than the large-amplitude first-order kernel. The remaining components approach zero rather quickly, an indication that, for this condition and for this motion (plunge), the first-order kernel may be sufficient for predicting nonlinear plunge responses.

Figure 10 is a comparison of two nonlinear sinusoidal plunge responses from CFL 3D and the convolved responses using the large-amplitude first-order kernel and including the addition of the $h_{0}$ term to the total response. The smaller, CFL3D response corresponds to a plunge amplitude of 0.01 (based on chord length). The larger CFL3D response corresponds to a plunge amplitude of 0.05 . The reduced frequency of the plunging motions is 0.67 . These results indicate that the first-order kernel can be used to accurately predict the nonlinear plunge responses of this CFL3D model over a wide range of amplitudes. Of great importance is the fact that the first-order kernel, which has a temporal duration of less than 20 time steps, can be used to predict the response of an input of arbitrary length (5000 time steps, in this case). This is due to the mathematical efficiency of convolution.

It is important to properly choose the amplitude used for identifying the first- and second-order kernels. One possible approach for determining this identification amplitude is to base it on 1) physical considerations and 2) code execution limitations. If the CFD code executes properly for the largest input amplitude of interest (a sinusoidal input, for example) and the input amplitude is physically realistic, then the accuracy and effectiveness of the first- and secondorder kernels, identified within this amplitude range, will be nearly optimal assuming convergence issues are satisfied.

\section{Computational Efficiency}

The cost of each sinusoidal plunge response using CFL3D was about 2,000 CPU seconds and a turnaround time of about a day. These responses were for a particular frequency of motion that required a particular length of time for a certain number of cycles. A change in the input (frequency, for example) requires another execution of the CFL3D code. This translates into large (and expensive) turnaround times due to: 1) the time spent waiting for job execution in the queue of a supercomputer, for example, and 2) the time spent in actual execution of the code. The latter becomes even more expensive if several cycles of a low-frequency response are desired.

On the other hand, the results presented in this paper show that the application of the Volterra theory to CFD codes reduces computational turnaround time significantly. This computational efficiency is achieved by virtue of the following: 1) the short duration of the first- and second-order kernels (see Figs. 8 and 9 ) and 2) the mathematical efficiency of convolution. The short duration of the kernels leads to very small turnaround times. In fact, the kernels presented here were generated using the debug queue of a Cray supercomputer. The debug queue is limited to no more than 300 time steps for the purpose of code debugging. The average turnaround time for the responses needed for computing the kernels was about five minutes. The computation of the first-order kernel, for the RAE airfoil using the CFL3D code, cost $400 \mathrm{CPU}$ seconds; 200 for each of the two required responses (Eq. (3)). Once the kernels were identified, costly re-execution of the CFD code was side-stepped by applying convolution for every new input of interest. The cost of each convolution, for the plunge motions investigated, was 30 seconds per motion on a workstation.

\section{CONCLUSIONS}

Reduced-order aerodynamic models based on linear and nonlinear aerodynamic impulse responses have been discussed. The autonomous (time-invariant) nature of the Navier-Stokes equations was described in detail and the applicability of the Volterra theory of nonlinear systems to the Navier-Stokes equations was formally presented. The nature and computational efficiency of linear and nonlinear discrete-time convolution was described as well.

The method was applied first to a nonlinear circuit described by the Riccati equation and then, to a plunging airfoil using the CFL3D (NavierStokes) flow solver with the Spalart-Allmaras turbulence model. Results presented include the linear and nonlinear impulse responses for these systems as a function of several parameters. These parameters range from equation coefficients (Riccatti circuit) to varying the amplitude of identification of the first-order kernels (CFL3D model). 
The nonlinear impulse responses capture the nonlinear nature of the system under investigation. Computational cost comparisons were presented for the CFL3D/RAE 2822 model. It was shown that Volterra kernels provide significant computational efficiency over the full (and repetitive) solution of the complete system (CFL3D).

\section{REFERENCES}

'Leishman, J.; and Crouse, G., "A State-Space Model of Unsteady Aerodynamics in a Compressible Flow for Flutter Analyses," AIAA Paper No. 89-0022, 27th Aerospace Sciences Meeting, January 9-12, 1989, Reno, Nevada.

'Nixon, D., "Alternative Methods for Modeling Unsteady Transonic Flows, Unsteady Transonic Aerodynamics, Vol. 120 of Progress in Astronautics and Aeronautics, Ed. by D. Nixon, AIAA, 1989.

${ }^{3}$ Reisenthel, P.H., "Development of a Nonlinear Indicial Model for Maneuvering Fighter Aircraft," AIAA Paper No. 96-0896, 34th Aerospace Sciences Meeting \& Exhibit, January 15-18, 1996, Reno, Nevada.

${ }^{4}$ Marques, F.; and Anderson, J., "Modelling and Identification of Non-Linear Unsteady Aerodynamic Loads by Neural Networks and Genetic Algorithms," Proceedings of the 20th International Council of the Aeronautical Sciences, Sorrento, Italy, 8-13 September, 1996, Volume 1, ICAS-96-7.1.1, pp 243-251.

${ }^{5}$ Dowell, E.H.; Hall, K. C.; and Romanowski, M. C.: "Eigenmode Analysis in Unsteady Aerodynamics: Reduced Order Models," ASME Applied Mechanics Review, Volume 50, Number 6, June 1997, pp371-386.

'Baker, M.L., "Model Reduction of Large, Sparse, Discrete Time Systems with Application to Unsteady Aerodynamics," PhD Dissertation, University of California at Los Angeles, 1996.

'Stalford, H.; Baumann, W. T. ; Garrett, F. E. ; and Herdman, T. L. : "Accurate Modeling of Nonlinear Systems Using Volterra Series Submodels," Presented at the 1987 American
Control Conference, Minneapolis, MN, June 1012, 1987.

${ }^{8}$ Rodriguez, E.A., "Linear and Nonlinear Discrete-Time State-Space Modeling of Dynamic Systems for Control Applications," $\mathrm{PhD}$ Dissertation, Purdue University, December 1993.

9'Silva, W.A., "Application of Nonlinear Systems Theory to Transonic Unsteady Aerodynamic Responses," Joumal of Aircraft, Volume 30, Number 5, September-October 1993, pp. 660-668.

${ }^{10}$ Silva, W.A., "Extension of a Nonlinear Systems Theory to General-Frequency Unsteady Transonic Aerodynamic Responses," AIAA Paper No. 93-1590, Presented at the 34th Structures, Structural Dynamics, and Materials Conference, La Jolla, California, April 19-21, 1993.

"Silva, W.A., "Identification of Linear and Nonlinear Aerodynamic Impulse Responses Using Digital Filter Techniques," AlAA Paper No. 97-3712, Presented at the AIAA Atmospheric Flight Mechanics Conference, August 11-13, 1997, New Orleans, LA.

${ }^{12}$ Silva, W. A., "Discrete-Time Linear and Nonlinear Aerodynamic Impulse Responses for Efficient CFD Analyses," PhD dissertation, College of William \& Mary, December 1997.

${ }^{13}$ Khalil, H.K., Nonlinear Systems, Macmillan Publishing Company, New York, 1992.

${ }^{14}$ Bisplinghoff, R. L.; and Ashley, H., Principles of Aeroelasticity, Dover Publications, Inc., New York, 1975.

${ }^{15} \mathrm{Ku}, \mathrm{Y} . \mathrm{H}$.; and Su, C. C.: "Volterra Functional Analysis of Nonlinear Time-Varying Systems," Joumal of the Franklin Institute, Volume 204, Number 6, December 1967.

${ }^{16}$ Volterra, V.: Theory of Functionals and of Integral and Integro-Differential Equations, Dover Publications, Inc., New York, 1959.

${ }^{17}$ Rugh, Wilson J. : Nonlinear System Theory The Volterra-Wiener Approach. The Johns Hopkins University Press, 1981. 
${ }^{18}$ Schetzen, Martin : The Volterra and Wiener Theories of Nonlinear Systems, John Wiley \& Sons, 1980.

"Boyd, S.P.: "Volterra Series: Engineering Fundamentals," PhD Dissertation, University of California, Berkeley, 1985.

2"Wiener, N.: "Response of a Nonlinear Device to Noise," dissertation No. 129, Radiation Laboratory, M.I.T., Cambridge, Massachusetts, April 1942. (Also published as U.S. Department of Commerce Publications PB-58087.)

${ }^{21}$ Lee, Y.W.; and Schetzen, M.: "Measurement of the Wiener Kernels of a Non-linear System by Crosscorrelation," Intemational Joumal of Control, Volume 2(3), pp. 237-254, September 1965.

${ }^{22}$ Flake, R. H., "Volterra Series Representation of Nonlinear Systems," Transactions of the American Institute of Electrical Engineers, Volume 81, 1962, Part II: Applications and Industry.

${ }^{23} \mathrm{Ibrahim}, \quad$ R. A., "Response Analysis of Nonlinear Systems Using FunctionalPerturbational Type Approach," 6th International Modal Analysis Conference, February 1-4, 1988, Kissimmee, FL.

${ }^{24}$ Boyd, S. ; Chua, L. O. ; and Desoer, C. A. : Analytical Foundations of Volterra Series, IMA Journal of Mathematical Control \& Information, 1984, Volume 1, pp. 243-282.

${ }^{25}$ Brilliant, M. B.: "Theory of the Analysis of Nonlinear Systems," Technical dissertation 345, M.I.T. Research Laboratory of Electronics, March 1958.

${ }^{26}$ Boyd, S. P.; and Chua, L. O.: "Fading Memory and the Problem of Approximating Nonlinear Operators with Volterra Series" IEEE Transactions on Circuits and Systems, Volume CAS-32, Number 11, November 1985.

${ }^{27} \mathrm{Ku}, \mathrm{Y}$. H.; and Wolf, A. A.: "Volterra-Wiener Functionals for the Analysis of Nonlinear Systems," Joumal of the Franklin Institute, Volume 281, Number 1, January 1966.
${ }^{28}$ Bedrosian, E.; and Rice, S. O.: "The Output Properties of Volterra Systems (Nonlinear Systems with Memory) Driven by Harmonic and Gaussian Inputs," Proceedings of the IEEE, Volume 59, Number 12, December 1971.

${ }^{2 y}$ Sandberg, I. W.: "Nonlinear Input-Output Maps and Approximate Representations," AT\&T Technical Journal, Volume 64, Number 8, October 1985, pp. 1967-1983.

${ }^{30}$ Bendat, J. S., Nonlinear System Analysis \& Identification from Random Data, A WileyInterscience Publication, 1990.

${ }^{31}$ Tryon, P. V., "The Bispectrum and HigherOrder Spectra: A Bibliography," National Bureau of Standards Technical Note 1036, April 1981.

${ }^{32}$ Nikias, C. L.; and Petropulu, A. P., HigherOrder Spectra Analysis: A Nonlinear Signal Processing Framework. Prentice-Hall Signal Processing Series, Alan V. Oppenheim, Series Editor, 1993.

${ }^{33}$ Boyd, Stephen ; Tang, Y. S. ; and Chua, Leon A. : "Measuring Volterra Kernels," IEEE Transactions on Circuits and Systems, Volume CAS-30, Number 8, August 1983.

${ }^{34}$ Ritz, C. P.; and Powers, E. J., "Estimation of Nonlinear Transfer Functions for Fully Developed Turbulence," Physica D 'Nonlinear Phenomena', May 1986, pp320-334.

${ }^{35}$ Roberts, J. B., "Nonlinear Analysis of Slow Drift Oscillations of Moored Vessels in Random Seas," Journal of Ship Research, Volume 25, Number 2, June 1981, pp. 130-140.

${ }^{36}$ Krist, S.L.; Biedron, R.T.; and Rumsey, C.L., "CFL3D User's Manual (Version 5.0)," November 1996, First Edition.

${ }^{37}$ Rumsey, C. L.; Sanetrik, M. D.; Biedron, R. T.; Melson, N. D.; and Parlette, E. B., "Efficiency and Accuracy of Time-Accurate Turbulent Navier-Stokes Computations," AIAA Paper No. 95-1835, Presented at the 13th ALAA Applied Aerodynamics Conference, June 19-22, 1995, San Diego, CA. 


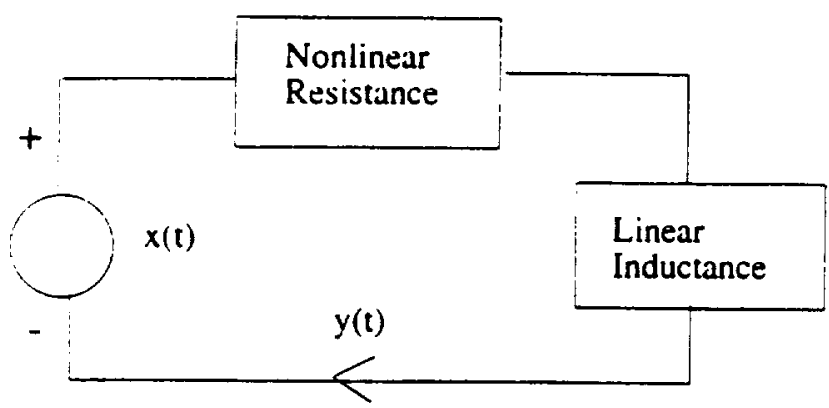

Fig ! Simple nonlinear circuit defined by the Riccati equation with $x(t)$ (voltage) as input and $y(t)$ (current)

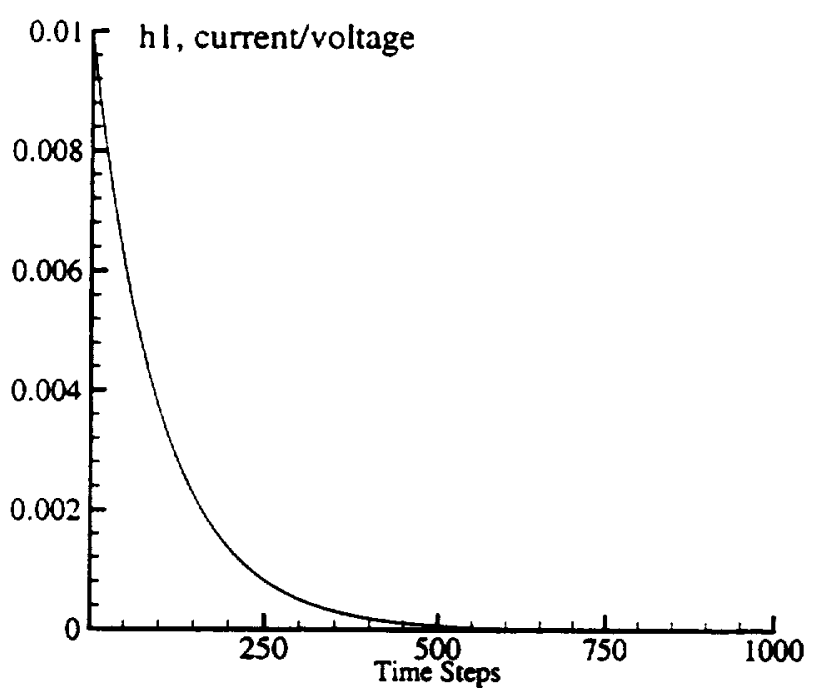

Fig 2 First-order kemel for the Riccati nonlinear circuit, Case 1 .

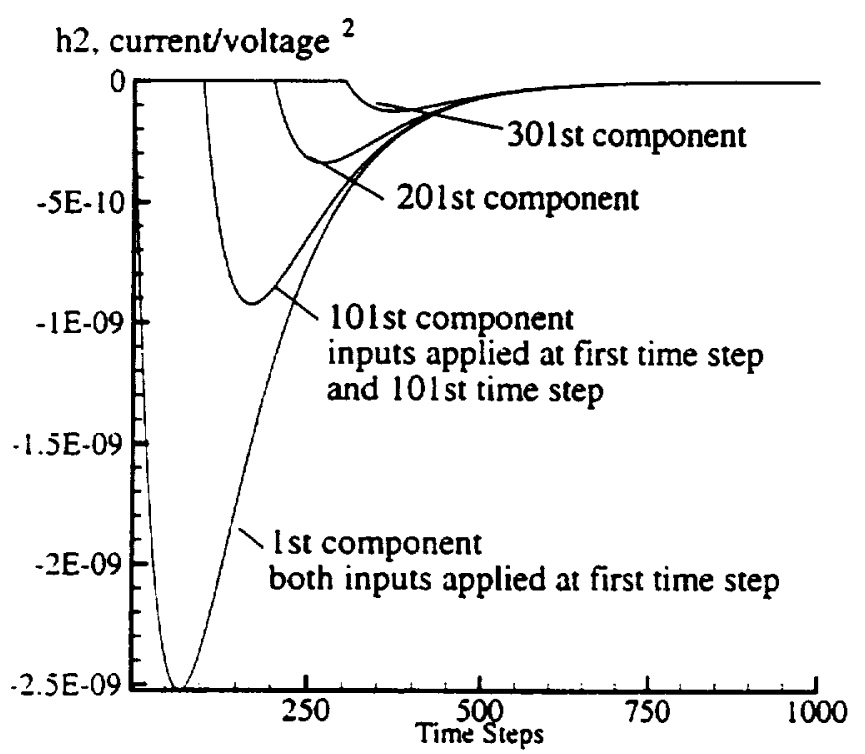

Fig 3 Selected components of the second-order kemel for the Riccati nonlinear circuit, Case 1.

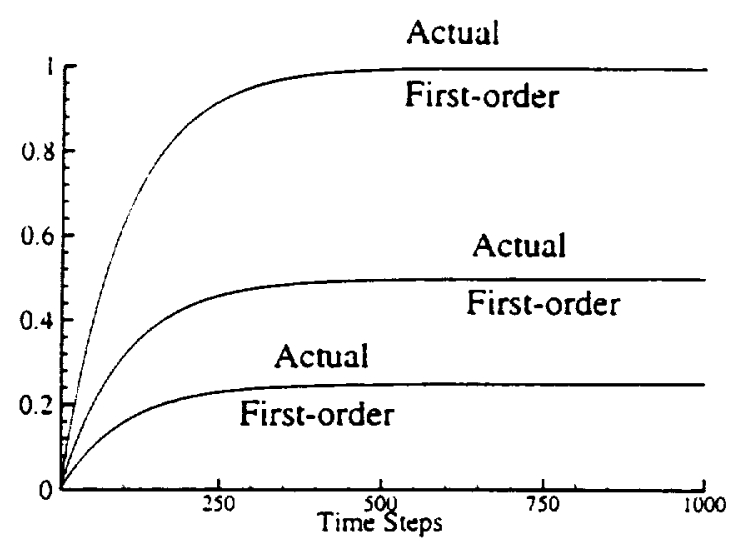

Fig 4 Comparison of actual and first-order step

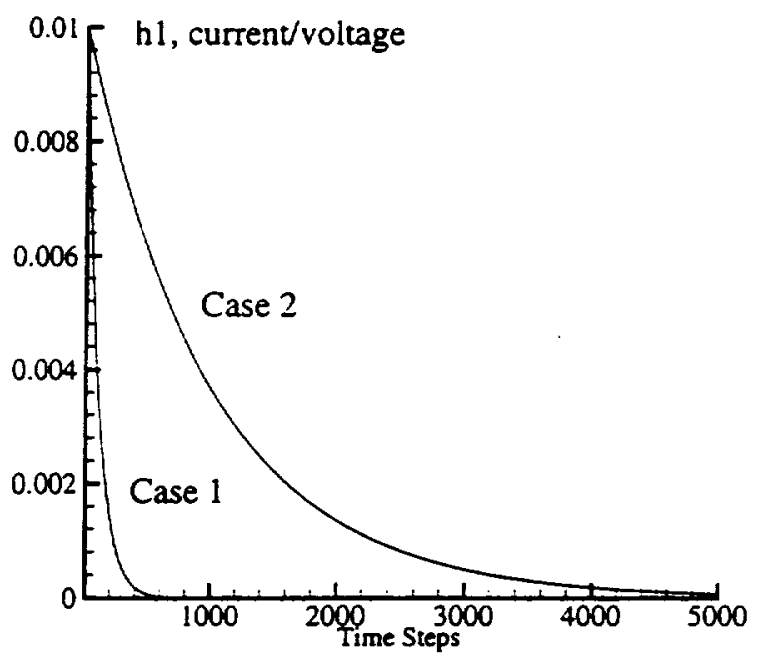

Fig 5 First-order kemels for Riccati nonlinear circuit, Case 1 and Case 2.

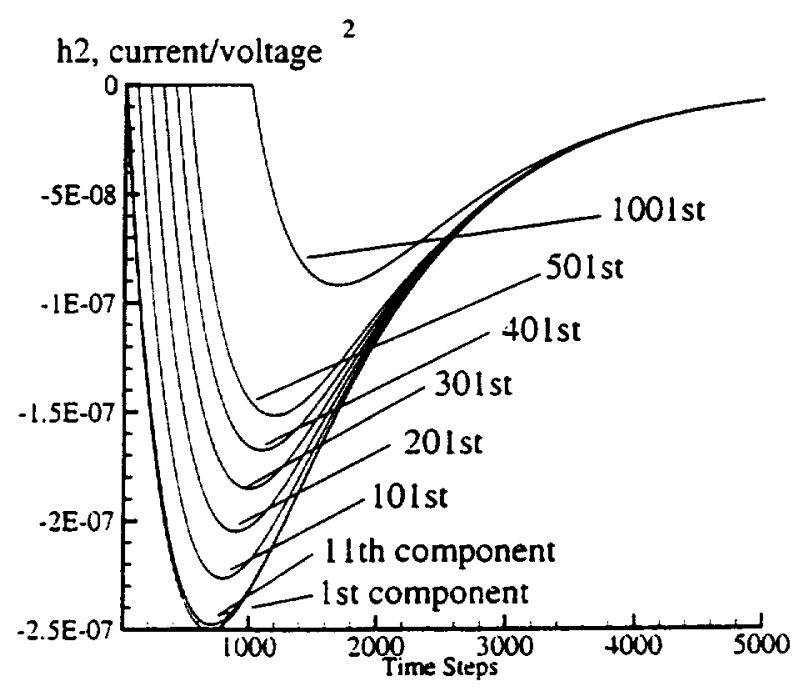

Fig 6 Selected components of the second-order kemel for the Riccati nonlinear circuit, Case 2. 


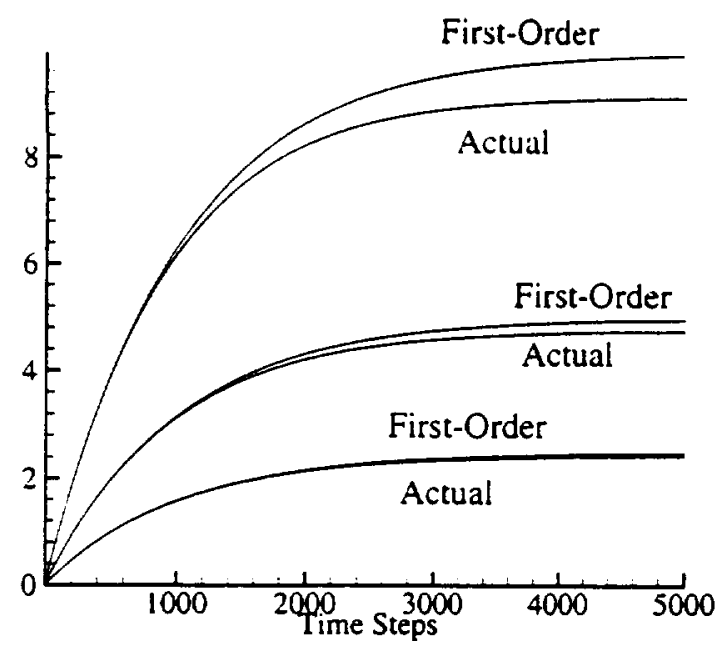

Fig 7 Comparison of actual and first-order step responses for the Riccati nonlinear circuit, Case 2.

Normal Force/Plunge

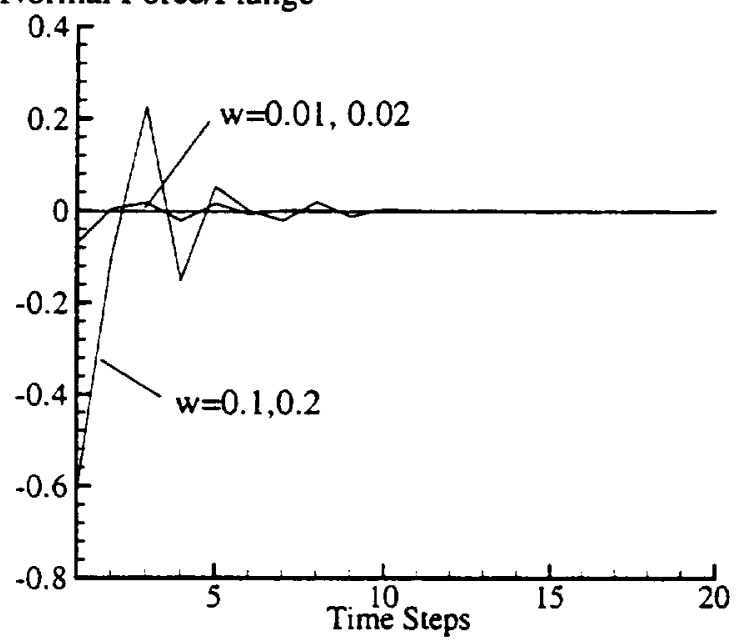

Fig 8 First-order kernels for RAE airfoil in plunge, effect of identification amplitudes.

Normal Force/Plunge

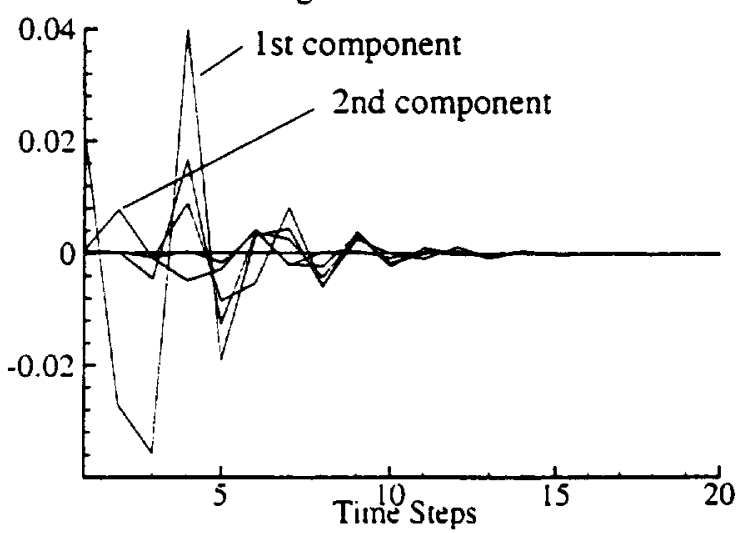

Fig 9 First live components of the second-order kernel for the RAE airfoil in plunge, largest ID amplitude.
Normal Force

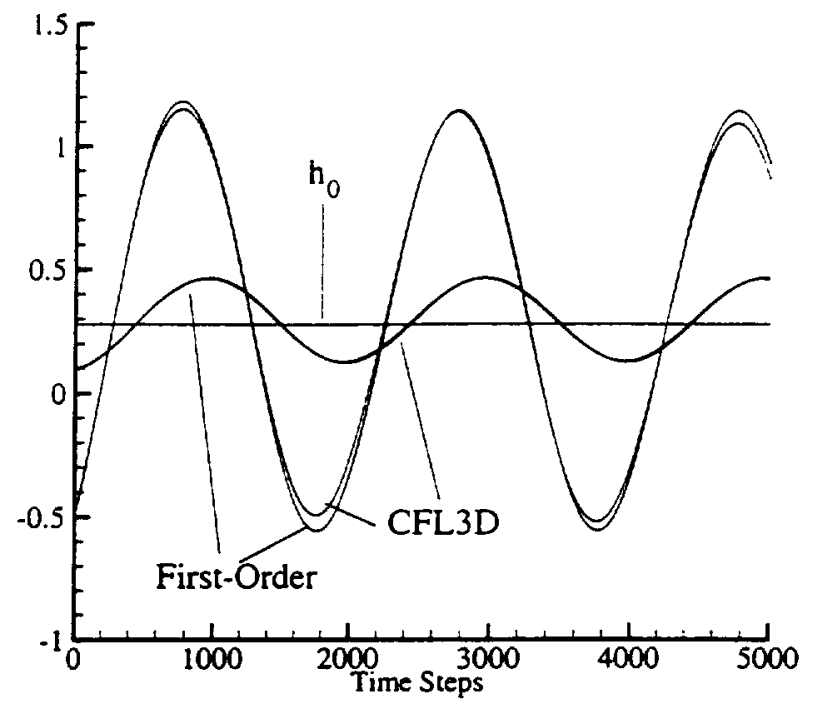

Fig 10 Comparison of CFL3D and first-order responses for two plunge amplitudes $(0.01,0.05)$. 
